Henrik Hautzinger

Der Ruf von Branchen 
GABLER RESEARCH 
Henrik Hautzinger

\section{Der Ruf von Branchen}

Eine empirische Untersuchung

zur Messung, Wechselwirkung

und Handlungsrelevanz

der Branchenreputation

Mit einem Geleitwort von Prof. Dr. Sabrina Helm 
Bibliografische Information der Deutschen Nationalbibliothek

Die Deutsche Nationalbibliothek verzeichnet diese Publikation in der

Deutschen Nationalbibliografie; detaillierte bibliografische Daten sind im Internet über $<$ http://dnb.d-nb.de> abrufbar.

Dissertation Private Universität Witten/Herdecke, 2008

1. Auflage 2009

Alle Rechte vorbehalten

(c) Gabler | GWV Fachverlage GmbH, Wiesbaden 2009

Lektorat: Claudia Jeske | Viktoria Steiner

Gabler ist Teil der Fachverlagsgruppe Springer Science+Business Media. www.gabler.de

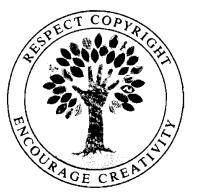

Das Werk einschließlich aller seiner Teile ist urheberrechtlich geschützt. Jede Verwertung außerhalb der engen Grenzen des Urheberrechtsgesetzes ist ohne Zustimmung des Verlags unzulässig und strafbar. Das gilt insbesondere für Vervielfältigungen, Übersetzungen, Mikroverfilmungen und die Einspeicherung und Verarbeitung in elektronischen Systemen.

Die Wiedergabe von Gebrauchsnamen, Handelsnamen, Warenbezeichnungen usw. in diesem Werk berechtigt auch ohne besondere Kennzeichnung nicht zu der Annahme, dass solche Namen im Sinne der Warenzeichen- und Markenschutz-Gesetzgebung als frei zu betrachten wären und daher von jedermann benutzt werden dürften.

Umschlaggestaltung: KünkelLopka Medienentwicklung, Heidelberg Gedruckt auf säurefreiem und chlorfrei gebleichtem Papier Printed in Germany 


\section{Geleitwort}

„Mitgefangen, mitgehangen?“ - Die vorliegende Arbeit ist dem Zusammenspiel von Branchenreputation und Unternehmensreputation gewidmet. Sie stellt damit die erste umfassende Analyse eines Themenfeldes dar, das aus Sicht des Reputationsmanagements höchst bedeutsam ist. Schließlich stellt das produktionsbezogene Wirkungsfeld eines Unternehmens zumindest kurz- und zumeist auch mittelfristig ein Datum dar und Unternehmen müssen sich mit dem Ruf ihrer Branche isoliert oder kooperativ auseinandersetzen.

Die Reputation von Unternehmen ist eines der wesentlichen immateriellen „Assets“, dessen Erfolgsbeitrag weder in der Praxis noch Theorie bestritten wird. Henrik Hautzinger betrachtet in seiner Arbeit die möglichen Wechselwirkungen zwischen Branchen- und Unternehmensruf auf Grundlage von Informations- und Industrieökonomik. Jedes Unternehmen ist in eine oder mehrere Branchen eingebettet, die ebenfalls über einen Ruf verfügen. Das Zusammenspiel zwischen Branchen- und Unternehmensruf ist dann besonders wichtig, wenn die Entwicklung der Reputation eines Unternehmens verstanden und gegebenenfalls auch gesteuert werden soll. Henrik Hautzinger arbeitet heraus, dass der Branchenruf eine entscheidende Determinante im Wettbewerb zwischen Unternehmen ist und - anders als früher nicht nur die „üblich verdächtigen“ Branchen wie Öl- und Tabakindustrie im medialen und Verbraucherinteresse stehen. Zudem finden sich alle Unternehmen auf bestimmten Märkten wie dem Arbeits- und Kapitalmarkt als Wettbewerber wieder, was die Relevanz der bearbeiteten Thematik unterstreicht.

Henrik Hautzinger entwickelt den ersten umfassenden Messansatz für den Branchenruf. In einer explorativen empirischen Studie kann er belegen, dass es tatsächlich Interdependenzen zwischen den beiden Reputationen gibt und, wichtiger noch, dass aus Sicht verschiedener Unternehmen die Wirkstrukturen unterschiedlich ausgeprägt sein können. Manche Unternehmen formen den Ruf ihrer Branche, manche Branchen drücken einem Unternehmen das Stigma der Industrie auf. Henrik Hautzinger geht jedoch noch einen entscheidenden Schritt weiter und untersucht den Einfluss des Branchenrufs auf Entscheidungen auf Märkten. In einer weiteren empirischen Studie kann er aufzeigen, dass Bewerber auf dem Arbeitsmarkt 
tatsächlich vom Ruf der Branche eines potenziellen Arbeitgebers in ihrer Bewerbungsabsicht beeinflusst werden und dass für manche Branchen dieser Einfluss entscheidender ist als der Ruf des Unternehmens selbst. Zentral ist also die Erkenntnis, dass mit besserem Branchenruf die Bewerbungsabsicht für die Branche steigt. Und je besser der Ruf einer Branche von potenziellen Bewerbern wahrgenommen wird, desto besser ist auch der Ruf der Unternehmen aus der Branche. Der Branchenruf ist für jedes Unternehmen relevant, denn Bewerber fällen inre Bewerbungsentscheidung für einen Arbeitgeber vor dem Hintergrund der Entwicklung des Branchenrufs. Je mehr potenzielle Bewerber dabei über ein Unternehmen bzw. über eine Branche wissen, desto mehr Bedeutung kommt dem Ruf bei ihrer Bewerbungsentscheidung zu.

Der Ruf einer Branche lässt sich auf Branchen- wie auch auf Unternehmensebene managen. Der Verfasser empfiehlt, dass Unternehmen beim Management ihres Rufs potenzielle Gefahren, die von Seiten der Branche drohen, vorab identifizieren und Gegenstrategien bereit halten sollten. Unternehmen aus Branchen mit gutem Ruf müssen diesen Vorteil bei der Bewerbergewinnung betonen. Bei einem schlechten Branchenruf gilt es ein attraktives Gegengewicht aufzubauen.

Damit legt Henrik Hautzinger einen soliden Entwurf für die Integration des Branchenrufs in das Reputationsmanagement vor, der für die wissenschaftliche wie praktische Auseinandersetzung Bedeutung hat. Die Lektüre seiner Dissertationsschrift ist damit für Studierende und Wissenschaftler mit einem Bezug zum Reputationsmanagement empfehlenswert. Insbesondere sei sie jedoch jenen Managern ans Herz gelegt, die dem Reputationsmalus ihrer Industrie entkommen oder die umgekehrt von einem Reputationsbonus ihrer Branche profitieren möchten.

Professor Dr. Sabrina Helm 


\section{Vorwort}

Kaum ein ökonomisch relevanter Vermögensgegenstand konnte in den letzten Jahren einen größeren Anstieg in der Aufmerksamkeit verzeichnen als der Ruf. Vor allem bei Branchen finden sich feste, in der Gesellschaft etablierte Wahrnehmungen hinsichtlich deren Reputation. Diese Wahrnehmungen beeinflussen maßgeblich die ökonomische Entwicklung einer Branche und ihrer Mitglieder. Als Forschungsgegenstand wurde der Branchenruf jedoch bislang stark vernachlässigt. Mit der vorliegenden Arbeit ist daher das Ziel verbunden, grundlegende Einsichten in den Ruf von Branchen zu gewinnen. Neben der erstmaligen Messung des Branchenrufs werden daher auch die Wechselbeziehungen mit dem Ruf der Unternehmen aus der Branche beleuchtet. Praxisrelevant sind vor allem die Ergebnisse der empirischen Untersuchung zur Handlungsrelevanz des Branchenrufs bei Bewerbern.

Am Gelingen eines Dissertationsprojekts sind stets zahlreiche Personen beteiligt. Ganz besonderer Dank geht natürlich an meine akademische Lehrerin Frau Professor Dr. Sabrina Helm. Ihre Kompetenz und Freude an der Thematik war ein großer Ansporn und hat maßgeblich dazu beigetragen, dass es mir viel Spaß gemacht hat die vorliegende Arbeit zu verfassen. Frau Professor Dr. Friederike Wall (Universität Witten/Herdecke) danke ich vielmals für die spontane Übernahme des Zweitgutachtens. Herr Professor Dr. Bernd Günter (Universität Düsseldorf) hat unter starken zeitlichen Restriktionen das Drittgutachten verfasst, hierfür ebenfalls meinen besten Dank.

Dass ich mit der Dissertationszeit sehr viele schöne Erinnerungen verbinde, liegt aber auch an dem tollen Umfeld, in dem ich arbeiten konnte. Neben dem fachlichen Austausch ist für die Motivation vor allem auch die gedankliche Ablenkung abseits der Diss entscheidend. Auf tatkräftige Unterstützung in beiden Belangen konnte ich mich stets verlassen. Den nachfolgenden Personen gilt daher mein ganz persönlicher Dank:

Julia Spelsiek und Christian Klode als Reputationsexperten und motivierende Gesprächspartner vom Marketing Lehrstuhl an der Universität Witten/Herdecke, 
Christian Langmann als treuer Telefon-Kontaktmann für vertiefte PLS-Diskussionen an der Helmut-Schmidt-Universität in Hamburg, Timo Reister und Kollegen vom ZEW als zuverlässige Freizeitgestalter in Mannheim und Umgebung, Carsten Höhn für Treffen im Raum Frankfurt, Jana Prigge vom Lehrstuhl Homburg für Abendessen an der Uni in Mannheim sowie Alexander Karl für ausgedehnte Wochenendaktivitäten in Heilbronn. Auch meine Schwester Nina Schwab-Hautzinger mit Ihrer Familie in Basel war eine verlässliche Ansprechpartnerin in der Diss-Zeit.

Ohne meine Eltern Isolde und Heinz Hautzinger wäre die vorliegende Arbeit allerdings nicht möglich gewesen. Auf Ihren Rückhalt, Ihre Unterstützung konnte und kann ich mich jederzeit verlassen. Vielen herzlichen Dank!

Henrik Hautzinger 


\section{Inhaltsverzeichnis}

Abbildungsverzeichnis ....................................................................... XV

Tabellenverzeichnis ................................................................................... XVII

Abkürzungsverzeichnis …..................................................................... XIX

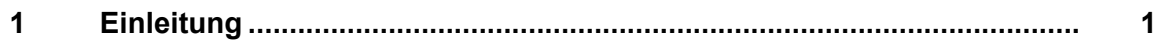

1.1 Die Relevanz des Branchen- und Unternehmensrufs ...................... 1

1.2 Forschungsfragen und Eingrenzung der Untersuchung.................. 6

1.3 Aufbau der Arbeit ............................................................................. 9

2 Definition, Entstehung und Wirkung der Rufkonstrukte......................... 13

2.1 Der Ruf von Unternehmen............................................................ 13

2.1.1 Definition von Unternehmensruf............................................ 13

2.1.2 Corporate Image, Corporate Identity und Corporate Brand Abgrenzung der Corporate Reputation von verwandten Konstrukten

2.1.3 Identifikation von ökonomischen und vorökonomischen Treibern des Unternehmensrufs

2.1.4 Branchenmerkmale als indirekte Treiber des Unternehmensrufs

2.2 Der Ruf von Branchen ............................................................. 28

2.2.1 Definition und Klassifikation von Branchen .............................. 28

2.2.2 Identifikation der Branche als Rufträger ................................. $\quad 30$

2.2.3 Definition von Branchenruf .................................................... 32

2.3 Die Rolle der Stakeholder bei der Rufbildung ................................. 34

2.3.1 Grundlegende Stakeholderansätze zur Erklärung der

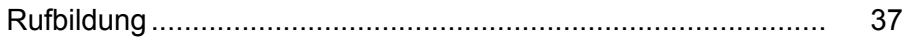

2.3.2 Spezifische Stakeholderaspekte im Kontext von Reputation ..... 39

2.4 Der Zusammenhang zwischen Ruf und unternehmerischen Zielgrößen

2.4.1 Strategische Zielgrößen einer Unternehmung und Auswirkungen des Rufs 
2.4.2 Das Wirkungsgefüge von Ruf und unternehmerischen

Zielgrößen

3 Die Messung der Rufkonstrukte.

3.1 Generelle Aspekte und Determinanten der Rufmessung ................ 49

3.2 Verschiedene Ansätze zur Messung des Unternehmensrufs ........ 54

3.2.1 America's Most AdmiRed Companies des Fortune Magazin .... 54

3.2.2 Die Imageprofile des MANAGER-MAGAZINS ............................... 56

3.2.3 Der Global RepTrak ${ }^{\text {TM }}$ Pulse des Reputation InStitute ......... 57

3.2.4 Der Unternehmensruf nach HELM (2007a) ............................ 59

3.2.4.1Charakteristika und Indikatoren des Messmodells ......... 59

3.2.4.2Kritische Würdigung des Messansatzes........................ 62

3.3 Der Stand zur Messung des Branchenrufs .................................... 63

3.3.1 Industrieökonomische Aspekte zur Messung des

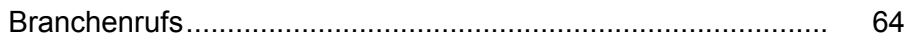

3.3.2 FomBRUNs (1996), reputational capital' von Branchen............... 65

3.3.3 Messansätze für das Branchenimage ..................................... 66

3.4 Fazit zur Messung des Branchen- und Unternehmensrufs ............. 68

$4 \quad$ Wechselwirkungen zwischen Branchen- und Unternehmensruf ........... 69

4.1 Der Einfluss des Branchenrufs auf den Ruf einer Unternehmung........................................................................ 70

4.1.1 Wirkungen des Branchenrufs auf ein nicht-diversifiziertes Unternehmen

4.1.2 Wirkungen des Branchenrufs auf ein diversifiziertes Unternehmen

4.2 Der Einfluss des Unternehmensrufs auf den Ruf einer Branche....

4.2.1 Möglichkeiten und Grenzen der Einflussnahme auf den Branchenruf durch ein einzelnes Unternehmen

4.2.2 Möglichkeiten und Grenzen der Einflussnahme auf den Branchenruf durch eine Gruppe von Unternehmen.

4.3 Das Wirkungsgefüge von Branchen- und Unternehmensruf

4.3.1 Erklärungsansätze für die Wirkungsrichtungen der Wechselbeziehung

4.3.2 Erklärungsansätze zur Wahrscheinlichkeit, Stärke und Relationen der Wechselbeziehungen 
4.4 Erste empirische Erkenntnisse zur Wechselbeziehung zwischen dem Branchen- und Unternehmensruf.

4.4.1 Herleitung und Darstellung der Fragestellung zur Wechselbeziehung.

4.4.2 Ausgestaltung der empirischen Untersuchung zur konzeptionellen Darstellung der Wechselbeziehungen zwischen den Reputationen

4.4.3 Darstellung und Diskussion der Ergebnisse zur direkten Abfrage der Wechselbeziehung

$5 \quad$ Konzeptionelle Betrachtung der Branchen- und Unternehmensreputation

5.1 Neue Institutionenökonomik als Bezugsrahmen

5.1.1 Die Principial-Agent-Theorie

5.1.1.1Darstellung der Principal-Agent-Problematik.

5.1.1.2Reputation als impliziter Mechanismus in der Principial-Agent-Theorie.

5.1.2 Die Informationsökonomie als Teilbereich der Neuen Institutionenökonomik

5.1.2.1Unsicherheit über die Qualität von Gütern

5.1.2.2Abbau von Qualitätsunsicherheit durch Reputation

5.2 Neue Industrieökonomik als Bezugsrahmen

5.2.1 Reputation als Ressource

5.2.2 Der Branchenruf als Common-Pool-Ressource

5.2.3 Reputation als Eintrittsbarriere in eine Branche

5.2.4 Reputation als Mobilitätsbarriere zwischen Strategischen Gruppen in einer Branche

5.3 Fazit zur konzeptionellen Betrachtung.

6 Die Handlungsrelevanz des Branchenrufs am Beispiel von potentiellen Bewerbern

6.1 Die Branche und potentielle Bewerber

6.1.1 Bedeutung von potentiellen Bewerbern als Stakeholder

6.1.2 Identifikation der Branche im Kontext der Arbeitgebersuche......

6.2 Determinanten der Entscheidungsfindung von Bewerbern

6.2.1 Rolle und Bedeutung des Branchenrufs bei der Bewerbungsentscheidung 
6.2.2 Rolle und Bedeutung des Unternehmensrufs bei der Bewerbungsentscheidung

6.2.3 Rolle und Bedeutung der Arbeitgeberattraktivität bei der Bewerbungsentscheidung

6.3 Determinanten der Informationsverarbeitung bei Bewerbern.

6.3.1 Involvement bei der Arbeitsplatzsuche

6.3.2 Wissen über Branche und Unternehmen

6.4 Zusammenfassung der Hypothesen

$7 \quad$ Empirische Untersuchung zur Handlungsrelevanz des

Branchenrufs bei potentiellen Bewerbern.

7.1 Spezifikation des Strukturgleichungsmodells.

7.1.1 Das Grundmodell

7.1.2 Das erweiterte Modell

7.2 Grundlagen der empirischen Untersuchung

7.2.1 Untersuchungssubjekte und Untersuchungsgegenstände

7.2.1.1Potentielle Bewerber als Untersuchungssubjekte

7.2.1.2Festlegung der in der Untersuchung betrachteten Branchen

7.2.1.3Festlegung der in der Untersuchung betrachteten Unternehmen

7.2.1.4Zusammenfassung und Wahl des Befragungsdesigns ...

7.2.2 Online-Befragung als Erhebungsmethode und Ablauf der Untersuchung

7.2.3 Rekrutierung der Teilnehmerstichprobe und Antwortverhalten

7.2.4 Soziodemographische Daten der Stichprobe und Analyse der Datengrundlage

7.3 Die Operationalisierung von theoretischen Konstrukten.

7.3.1 Die Entwicklung eines Messansatzes .....

7.3.2 Die epistemische Beziehung von latenten Variablen

7.3.2.1Formative und Reflektive Beziehung

7.3.2.2Zur Wahl der epistemischen Beziehung....

7.4 Die Messansätze der empirischen Untersuchung.

7.4.1 Der Ansatz zur Messung des Branchenrufs 198

7.4.1.1Definition des Konstrukts. 198 
7.4.1.2Itemgenerierung und Vorstudien

7.4.1.3Das Messmodell für Branchenreputation

7.4.1.4Abgrenzung des Branchenrufs vom Unternehmensruf und inhaltliche Besonderheiten

7.4.2 Der Ansatz zur Messung des Unternehmensrufs nach HELM (2007a)

7.4.3 Der Ansatz zur Messung der Bewerbungsabsicht.

7.4.4 Der Ansatz zur Messung der Arbeitgeberattraktivität Branche/Unternehmen

7.4.5 Determinanten der Einflussstärke

7.4.5.1Die Messung von Involvement

7.4.5.2Die Messung von Wissen.....

7.4.6 Die Skala zur Beurteilung der Items

7.5 Güteprüfung des Strukturgleichungsmodells mit dem PartialLeast-Squares(PLS)-Verfahren

7.5.1 PLS im Vergleich zur Kovarianzstrukturanalyse.

7.5.2 Grundlegende Richtlinien der Güteprüfung. 224

7.5.3 Gütekriterien und Güteprüfung auf Messmodellebene 226

7.5.3.1Die formativen Konstrukte 226

7.5.3.2Die reflektiven Konstrukte 234

7.5.4 Gütekriterien und Güteprüfung auf Strukturmodellebene 237

7.5.5 Gütekriterien und Güteprüfung der zentralen mediierenden Effekte

7.5.6 Gütekriterien und Güteprüfung der moderierenden Effekte des erweiterten Modells

7.6 Zusammenfassung und kritische Würdigung

7.6.1 Die grundlegenden Ergebnisse der Untersuchung. 249

7.6.2 Kritische Würdigung der Untersuchung..... 255

8 Implikationen für das Reputationsmanagement und weitere empirische Forschung

8.1 Implikationen für das Reputationsmanagement von Branchen.

8.1.1 Grundsätzliche Strategien für das Management der

Branchenreputation

8.1.2 Branchenstrategien für den Wettbewerb auf dem Bewerbermarkt 
8.2 Implikationen für das Reputationsmanagement bei

Unternehmen

8.2.1 Grundsätzliche Strategien für das Management der

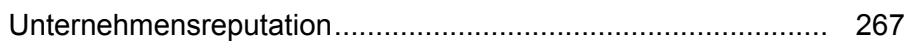

8.2.2 Unternehmensstrategien für den Wettbewerb auf dem Bewerbermarkt..................................................................... 268

8.3 Ansätze für weiterführende empirische Untersuchungen............... 270

8.4 Abschließendes Fazit ............................................................... 272

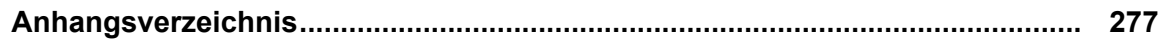

Anhang I: Fragebogen zur Wechselbeziehung ....................................... 277

Anhang II: Fragebogen zur Bewerberbefragung .................................. 281

Anhang III: Der Indikatorenpool zum Branchenruf................................. 288

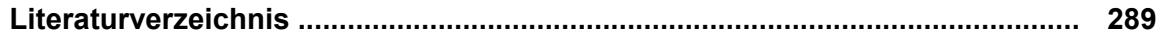




\section{Abbildungsverzeichnis}

Abbildung 1.1: Aufbau der Arbeit ....................................................... 11

Abbildung 2.1: Die Wahrnehmung der Anderen - Reputation als kollektives Konstrukt ................................................................. 19

Abbildung 2.2: $\quad$ Klassifikation der Stakeholder nach ihrer Relevanz ................. 36

Abbildung 4.1: $\quad$ Das Netzwerk der Images von DowLING .............................. 70

Abbildung 4.2: $\quad$ Die Identität der Branche und Unternehmen .......................... 93

Abbildung 4.3: Die möglichen Relationen von Branchen- und Firmenimages.... 98

Abbildung 4.4: Beispiel einer Reputationslandkarte einer Branche und ihrer Unternehmen ........................................................... 103

Abbildung 4.5: Die Reputationslandkarte der deutschen Automobilindustrie ..... 110

Abbildung 6.1: $\quad$ Die Lieblingsbranchen der BWL zum Berufseinstieg ................ 147

Abbildung 7.1: Das Grundmodell zur Handlungsrelevanz des Branchenrufs bei potentiellen Bewerbern........................................... 172

Abbildung 7.2: $\quad$ Das erweiterte Modell zur Handlungsrelevanz des Branchenrufs ......................................................... 173

Abbildung 7.3: Darstellung des Ablaufs der Online-Befragung ...................... 184

Abbildung 7.4: $\quad$ Reflektives Konstrukt mit drei Items .................................. 193

Abbildung 7.5: $\quad$ Formatives Konstrukt mit drei Items ................................ 195

Abbildung 7.6: Das Strukturgleichungsmodell mit Konstrukten und Anzahl der Indikatoren ...................................................... 222

Abbildung 7.7: $\quad$ Das Grundmodell mit den Ergebnissen der Kausalanalyse ....... 240

Abbildung 7.8: $\quad$ Grafische Darstellung einer mediierten Beziehung .................. 242

Abbildung 7.9: Grafische Darstellung eines Moderatoreffekts und der Interaktionsvariable 


\section{Tabellenverzeichnis}

Tabelle 2.1: $\quad$ Strategische Zielgrößen des Reputationsmanagements auf Unternehmensebene.

Tabelle 3.1: $\quad$ Die America's Most Admired Companies des (AMAC) des FORTUNE MAGAZINS

Tabelle 3.2:

Die Imageprofile des MANAGER-MAGAZINS

Tabelle 3.3:

Der REPTRAK ${ }^{\text {TM }}$ PULSE des Reputation Institutes

Tabelle 3.4:

Die 10 plus 1 Indikatoren für den Unternehmensrufs nach HELM.

Tabelle 4.1:

Szenarien der Reputation bei einer lateralen Diversifikation von Unternehmen.

Tabelle 4.2:

Die Rufkonstellationen zwischen Branche und Unternehmen....

Tabelle 4.3:

Untersuchungsgegenstände der empirischen Erhebung zur Wechselbeziehung der Reputationen

Tabelle 4.4:

Inhalte zur direkten Abfrage der Wechselwirkung zwischen den Reputationen

Tabelle 4.5:

Soziodemografische Eckdaten der Befragung zu den Wechselbeziehungen

Tabelle 5.1:

Die Typologisierung von Qualitätseigenschaften aus der Perspektive der Informationsökonomik

Tabelle 6.1:

Zusammenfassung der Hypothesen zur Relevanz des

Branchenrufs bei potentiellen Bewerbern.

Tabelle 7.1: $\quad$ Rangreihenfolge der Branchen gemäß den Ergebnissen der Frage „Wie ist Deiner Wahrnehmung nach der Ruf der nachfolgenden Branchen in der allgemeinen Öffentlichkeit?" ....

Tabelle 7.2: $\quad$ Unternehmensauswahl für die Untersuchung zur Handlungsrelevanz des Branchenrufs

Tabelle 7.3: $\quad$ Zusammenfassende Darstellung der Untersuchungssubjekte und -objekte der Bewerberbefragung......

Tabelle 7.4:

Das Antwortverhalten der Befragungsteilnehmer

Tabelle 7.5:

Beschreibung der Stichprobe: Alter und Status.

Tabelle 7.6:

Beschreibung der Teilnehmerstichprobe: Branche und Unternehmen

Tabelle 7.7:

Die 10 plus 1 Indikatoren für den Ruf von Branchen

Tabelle 7.8: Indikatoren zur Bewerbungsabsicht bei Branche und Unternehmen

Tabelle 7.9:

Merkmale zur Arbeitgerberattraktivität von Branchen und Unternehmen

Tabelle 7.10: Das Messmodell für Involvement 
Tabelle 7.12: Gütekriterien für die formativen Konstrukte Branchen- und Unternehmensruf

Tabelle 7.13: Gütekriterien für die formativen Konstrukte Arbeitgeberattraktivität Branche- und Unternehmen

Tabelle 7.14: Gütekriterien für das formative Konstrukt Involvement im erweiterten Modell.

Tabelle 7.15: Bewerbungsabsicht, Gütekriterien reflektive Konstrukte des ..... 236

Tabelle 7.16: Gütekriterien für die reflektiven Konstrukte Wissen über Branche und Unternehmen aus dem erweiterten Modell ........... 237

Tabelle 7.17: $\quad$ Gütekriterien auf der Ebene des Strukturmodells..................... 241

Tabelle 7.18: $\quad$ Gütekriterien und Signifikanz der Mediatorbeziehungen ............ 244

Tabelle 7.19: $\quad$ Stärke und Signifikanz der Moderatoren ................................. 249

Tabelle 7.20: $\quad$ Zusammenfassung der Ergebnisse der Hypothesenprüfung ..... 255 


\section{Abkürzungsverzeichnis}

\begin{tabular}{|c|c|}
\hline ADAC & Allgemeiner Deutscher Automobilclub \\
\hline AG & Aktiengesellschaft \\
\hline AICPA & American Institute of Certified Public Accountants \\
\hline AMAC & America's Most Admired Companies \\
\hline Anm. d. & Anmerkung des \\
\hline BMW & Bayerische Motorenwerke \\
\hline BWA & Bewerbungsabsicht(en) \\
\hline CA & Cronbachs alpha \\
\hline CEO & Chief Excecutive Officer \\
\hline CMA & Chemical Manufacturers Association \\
\hline CPR & Common-Pool-Resource \\
\hline CR & composite reliability (Konstruktreliabilität) \\
\hline DAX & Deutscher Aktienindex \\
\hline DBW & Die Betriebswirtschaft \\
\hline DDC & New York Diamond Dealer Club \\
\hline DEV & durchschnittlich erfasste Varianz \\
\hline ELM & Elaboration Likelihood Model \\
\hline EM & enviropreneurila marketing \\
\hline et al. & et alii \\
\hline f & folgende Seiten \\
\hline FF & Forschungsfrage \\
\hline $\mathrm{ff}$ & fortfolgende Seiten \\
\hline HGB & Handelsgesetzbuch \\
\hline
\end{tabular}




\begin{tabular}{|c|c|}
\hline $\mathrm{HHL}$ & Handelshochschule Leipzig \\
\hline Hrsg. & Herausgeber \\
\hline IAS & International Accounting Standards \\
\hline IFPMA & International Federation of Pharmaceutical Manufacturers \& \\
\hline & Associations \\
\hline INPO & Institute of Nuclear Power Operations \\
\hline i. S. & im Sinne \\
\hline Jg. & Jahrgang \\
\hline J\&J & Johnson \& Johnson \\
\hline LISREL & Linear Structural Relations \\
\hline n. r. & nicht relevant \\
\hline n. s. & nicht signifikant \\
\hline No. & number \\
\hline Nr. & Nummer \\
\hline o. V. & ohne Verfasserangaben \\
\hline PLS & Partial-Least-Squares \\
\hline PR & Public Relations \\
\hline $\mathrm{R}$ & reversed-coded \\
\hline rc & reputational capital \\
\hline $\mathrm{RQ}$ & Reputation Quotient \\
\hline S. & Seite \\
\hline SIC & US Standard Industrial Classification' \\
\hline SM & Schutzmarke \\
\hline SPSS & Statistical Package for the Social Science \\
\hline
\end{tabular}


TM

US

US-GAAP

u. U.

VAF

VDA

VIF

vgl.

Vol.

Wiwi

$w w w$

ZFP

ZfB

zfbf
Trademark

United States

United States Generally Accepted Accounting Principles

unter Umständen

variance accounted for

Verband der Automobilindustrie

variance inflation factor

vergleiche

volume

Wirtschaftswissenschaften

World Wide Web

Zeitschrift für Forschung und Praxis

Zeitschrift für Betriebswirtschaft

Schmalenbachs Zeitschrift für betriebswirtschaftliche Forschung 